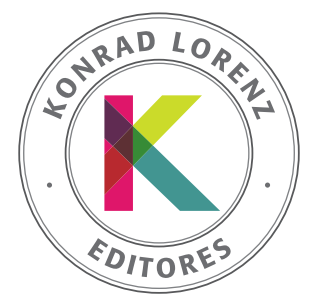

SUMA PSICOLÓGICA

http://editorial.konradlorenz.edu.co/suma-psicologica.html

\title{
Perfil de bienestar psicológico subjetivo en adolescentes uruguayos
}

\author{
María Eugenia Fernández Pintos*, Lilian R. Daset Carreto y Leandro Castelluccio
}

Facultad de Ciencias de la Salud, Departamento de Psicología Clínica y Salud, Universidad Católica del Uruguay, Montevideo, Uruguay

Recibido el 27 de junio de 2019; aceptado el 16 de octubre de 2019

\author{
PALABRAS CLAVE \\ Bienestar psicológico \\ subjetivo, \\ adolescentes, \\ índice de bienestar \\ personal, \\ factores de protección, \\ evaluación psicológica
}

\section{KEYWORDS}

Subjective wellbeing, adolescents, personal well-being index, protective factors, psychological assessment
Resumen El bienestar psicológico subjetivo (BPS) remite a la experiencia personal de cada sujeto con relación a la evaluación de la vida como un todo y por dimensiones. El estudio evalúa el BPS de 5189 adolescentes escolarizados uruguayos (capital e interior) de entre 12 a 21 años usando el índice de bienestar personal (PWI). Se realizaron análisis comparativos por sexo, lugar de residencia (capital e interior del país) y por grupos de edad, divididos en tres tramos. Los resultados indicaron que los adolescentes uruguayos tienen un nivel adecuado de BPS con relación a los promedios internacionales. En el análisis específico por dominios se observa que los adolescentes están más satisfechos con su pertenencia a los grupos que integra (conexión a la comunidad) y menos satisfechos con cuán seguros se sienten y su seguridad futura. En cuanto a diferencias por sexo, las chicas reportan un nivel significativamente menor de BPS en todos los dominios. No se encontraron diferencias significativas entre los estudiantes del interior y de la capital en su nivel de BPS en ninguno de los dominios. En cuanto a la comparación según tramos de edad, se observó una tendencia decreciente en el nivel de BPS desde los 15 años, que vuelve a subir en el tramo de adolescencia tardía (18 a 21). Estos resultados buscan aportar a estudios en proceso y brindan información para el diseño de políticas públicas e intervenciones educativas y clínicas con adolescentes escolarizados, que atiendan a factores de protección, además de las vulnerabilidades.

(C) 2019 Fundación Universitaria Konrad Lorenz. Este es un artículo Open Access bajo la licencia CC BY-NC-ND (http://creativecommons.org/licenses/bync-nd/4.0/).

\section{Subjective wellbeing profile of school going adolescents in Uruguay}

Abstract The subjective well-being (SWB) refers to the personal experience in relation to the evaluation of life as a whole and by dimensions. This study evaluates the SWB of 5189 Uruguayan school-going adolescents from 12 to 21 years old, using the Personal Wellbeing Index (PWI). Comparative analyzes were performed by sex, place of residence, and by age groups. Results indicated that Uruguayan adolescents have an adequate level of SWB. In the specific analysis by domains, it is observed that adolescents are more satisfied with their belonging groups in

* Autor para correspondencia.

Correo electrónico: maria.e.fp@gmail.com

http://dx.doi.org/10.14349/sumapsi.2019.v26.n2.6

0120-0534/@ 2019 Fundación Universitaria Konrad Lorenz. Este es un artículo Open Access bajo la licencia CC BY-NC-ND (http://creativecommons.org/licenses/by-nc-nd/4.0/). 
their community and less satisfied with how safe they feel and their future security. Regarding sex differences, girls report a significantly lower level of SWB in all domains. No significant differences were found between the students of the capital and countryside in their level of SWB in any of the domains. With regard to the comparison according to age groups, a decreasing trend was observed in the level of SWB from the age of 15, which rises again in the late adolescence segment (18 to 21). The results of the study contribute to previous studies and provide information for the design of public policies, educational and clinical interventions with school-going adolescents.

(c) 2019 Fundación Universitaria Konrad Lorenz. This is an open access article under the CC BYNC-ND license (http://creativecommons.org/licenses/bync-nd/4.0/).

El bienestar psicológico subjetivo (BPS) remite a la experiencia personal de cada sujeto con relación a la evaluación de la vida como un todo, a la vez que permite una mirada sobre las dimensiones que lo componen (Cummins, Eckersley, Pallant, Van Vugt \& Misajon, 2003; Diener, 2006). El bienestar psicológico es un constructo que incluye, por un lado, aspectos afectivos, que implican respuestas emocionales y, por otro, aspectos cognoscitivos, que tienen que ver con la satisfacción vital. Esta última expresa el juicio global de la persona sobre su vida (Diener, 2006, Rodríguez-Fernández et al., 2016). Además del juicio global, la satisfacción vital hace referencia a los diferentes ámbitos (i.e., familia, amigos, comunidad) y su evaluación específica en el momento presente (Huebner, Suldo \& McKnigth, Smith, 2004). El estudio del BPS aporta y complementa información desde el ámbito subjetivo, a los indicadores objetivos de la calidad de vida de las personas. Desde la perspectiva teórica de Cummins, el bienestar psicológico subjetivo se centra en la satisfacción con la vida; en el aspecto cognoscitivo. Cummins, con base en la evidencia empírica, propone la teoría homeostática del BPS, donde procesos neurológicos y psicológicos innatos operan para mantener un nivel positivo de bienestar (Cummins, Li, Wooden \& Stokes, 2014; Tomyn, Stokes, Cummins \& Dias, 2019). En condiciones normales, el nivel de BPS tiende a mantenerse con la ayuda de amortiguadores internos (i.e., optimismo, autoestima) y externos (i.e., relaciones, apoyo social) (Tomyn, Weinber \& Cummins, 2015).

La adolescencia es la etapa vital entre la pubertad y la adultez que implica multiplicidad de cambios y transiciones (Daset, López-Soler \& Hidalgo, 2009). El bienestar psicológico es un indicador de desarrollo de saludabilidad en esa etapa y, a la vez, un constructo que considera a los adolescentes como sujetos de derecho (Fernández, 2017; Huebner et al., 2004; Park, 2004); en tal sentido, es entonces importante contar con la percepción de los propios adolescentes sobre los distintos aspectos de su vida. La medida global de BPS a través del personal well-being index (PWI) ha demostrado cierta estabilidad en el tiempo y en las diferentes culturas (Lau, Cummins \& McPherson, 2005; The International Wellbeing Group, 2013). Sin embargo, dados los procesos biológicos y sociales experimentados en la etapa adolescente, estos podrían llegar a afectar el nivel de BPS (Cummins et al., 2014; García, Sagone, De Caroli \& Nima, 2017; GonzálezCarrasco et al., 2017; Tomyn et al., 2015); por tal razón, en este estudio se amplió el límite de edad de la muestra a los 21 años. En esta línea, el estudio de Rees y Dinisman (2015) compara la experiencia y evaluación sobre los diferentes aspectos de la vida de niños de 8 a 12 años de
11 países, encontrando variaciones significativas en el reporte de su bienestar en los diversos dominios, entre naciones y culturas. El reciente estudio de Casas y GonzálezCarrasco (2019), con datos de 15 países $(n=48040)$, evidenció el declive en el nivel de BPS a partir de los 10 años de edad para la mayoría de los países participantes y diferencias en el reporte del BPS referidas a la diversidad sociocultural. Por este motivo, es necesario contar con estudios adicionales que evalúen el BPS con bases nacionales y regionales y que incluyan un mayor rango de edades.

La investigación en el campo del bienestar psicológico subjetivo en adolescentes ha crecido en Latinoamérica. Sin embargo, en comparación con la acumulación de estudios con población adulta son de muy menor número (Fernández, 2017; Fernández et al., 2018; Ferreira-Lima \& Araújo de Morais, 2018; González-Carrasco et al., 2017; Rodríguez-Fernández et al., 2016; Schnettler et al., 2017).

La revisión bibliográfica sistemática sobre BPS en niños y adolescentes de Ferreira y Araújo (2018) identifica como principales ejes temáticos los estudios sobre bienestar subjetivo en los contextos educativos, familiares y comunitario, el BPS en relación con otras variables positivas (i.e., autoestima, optimismo, gratitud, afrontamiento), BPS según diferencias de género y culturales, el BPS con relación a la vulnerabilidad social y el estudio de las diversas medidas de evaluación del BPS.

Los antecedentes en Uruguay sobre BPS en adolescentes escolarizados refieren a un estudio con 325 jóvenes de un secundario en zona capitalina (Fernández et al., 2018). Las principales variables predictoras (sociodemográficas, de consumo de sustancias y psicopatología) indicaron que tres síndromes psicopatológicos se asociaron negativamente al BPS (depresión-ansiedad, ansiedad social y comportamiento disocial) y el síndrome que tiene como núcleo la resiliencia tuvo una asociación positiva. Los análisis del efecto moderador arrojaron que la edad, el sexo, la resiliencia y el uso de sustancias tuvieron interacciones significativas con el BPS -en uno y otro sentido-. Los resultados de este estudio indicaron que los adolescentes de la muestra se encontraban dentro de un rango adecuado de BPS (puntaje global) con relación a los estudios internacionales. En cuanto a las diferencias por género, las chicas presentaban un nivel significativamente menor de BPS en comparación con los varones. Estudios internacionales previos han mostrado también diferencias de género y edad en el nivel de BPS, con un nivel de reporte menor en las chicas y un declive hacia la adolescencia tardía (Brann et al., 2017; Casas et al., 2007; Castellá-Sarriera et al., 2012; González et al., 2017; Tomyn \& Cummins, 2011). Sin embargo, hay evidencia contradic- 
toria de resultados en cuanto a las diferencias en el nivel de BPS según el género (Castellá et al., 2012; Chen, Cai, He \& Fan, 2019; Ma \& Huebner, 2008; Strelhow, De Oliveira \& Câmara, 2010). Por tal motivo se hace necesario un análisis de dichas diferencias en estudios empíricos adicionales.

La relevancia del estudio radica en el aporte de evidencia empírica sobre el bienestar psicológico subjetivo en sus diversos dominios a la región $\mathrm{y}$, en especial, al contexto uruguayo. Este es el primer estudio en el país sobre el tema que cuenta con una base aleatoria nacional de adolescentes de instituciones educativas públicas y privadas de centros urbanos. Los resultados aportarían al desarrollo y evaluación de políticas públicas destinadas a adolescentes en el ámbito educativo y de salud y a los profesionales que trabajan con esta población.

El objetivo de este estudio es confeccionar un perfil nacional de bienestar psicológico subjetivo (BPS) de adolescentes escolarizados en Uruguay. Como objetivos específicos se plantearon la exploración de posibles diferencias por sexo, lugar de residencia (capital e interior) y por tramos de edad. Además, se llevará a cabo un análisis multidimensional específico de cada dominio del BPS, según el instrumento de índice de bienestar personal (personal well-being index, Cummins et al., 2003). Los dominios son: salud, nivel de vida, logros, seguridad (cuán seguro te sientes), conexión con la comunidad (grupos de los que forma parte), seguridad a futuro y relaciones con otros (pares y familia).

Basados en la revisión de la literatura y los resultados de los estudios antecedentes en nuestro país, nos planteamos las siguientes preguntas: ¿cuál es el nivel de BPS reportado de los adolescentes escolarizados uruguayos? ¿Existen diferencias con los hallazgos de los estudios internacionales de referencia? ¿Hay variaciones entre los reportes de BPS según el sexo y la edad en los adolescentes escolarizados uruguayos? ¿Se registran diferencias en el reporte del BPS según las regiones geográficas del país encuestadas? La primer hipótesis es que los adolescentes escolarizados uruguayos se encuentran dentro del rango esperado de niveles de bienestar, que se ubica en una media de 71 a 90 (The International Wellbeing Group, 2013). La segunda hipótesis, es que las chicas presentarán un nivel más bajo de BPS en comparación con los varones. Como tercera hipótesis en relación con la edad, los niveles de BPS serán más elevados en los estudiantes menores.

\section{Método}

\section{Participantes}

El total de participantes es de 5189 adolescentes, el $53.1 \%$ son chicas y el $46.9 \%$ varones. La muestra - seleccionada aleatoriamente- contó con estudiantes escolarizados de entre 12 a 21 años $(M=15.43$; $D E=1.77)$ de todo el país de centros educativos públicos y privados. Del total de la muestra, el $48.5 \%$ son de la capital y $51.5 \%$ del interior del país (pertenecientes a áreas pobladas que superan los 10000 habitantes). En cuanto a la distribución por ciclo escolar, el $42.4 \%$ cursaba segundo año del ciclo básico, el 33.3\% cuarto año del secundario (primero de bachillerato diversificado) y $24.3 \%$ cursaba sexto año (tercero de bachillerato diversificado). El sistema educativo de Uruguay está formado por un ciclo primario -con educación inicial incluida obligatoria a partir de los cuatro años- de dos niveles - dos años-, la primaria durante seis años y el secundario de seis años, tres básicos (hasta aquí todo es obligatorio) y tres con orientaciones a modo de bachillerato diversificado o escuelas técnicas.

\section{Procedimiento}

El estudio es de tipo transversal-correlacional. La selección de la muestra se basó en los registros administrativos sobre los centros educativos públicos y privados del país y se realizó en dos etapas. En la primera etapa, se seleccionaron 100 centros educativos con probabilidad proporcional al tamaño con base en la cantidad de estudiantes inscriptos. Se utilizó un diseño sistemático, ordenando los centros por tamaño. En la segunda etapa, a partir de los centros seleccionados en la primera fase, se seleccionan hasta tres grupos con igual probabilidad de selección, siguiendo el mismo diseño sistemático ordenando a los grupos por grado. Los tramos de edad considerados para realizar los análisis comparativos, fueron de 12 a 14, de 15 a 17 y de 18 a 21 años.

En el 2016, el Instituto Nacional de Estadística (INE) recabó los datos. Previa aprobación de la Administración de Educación Pública y los respectivos institutos; posteriormente, se informó sobre aspectos éticos y sobre los formularios que fueron completados en cada centro educativo en formato papel por los jóvenes. Se resguardó la confidencialidad y se trató la información acorde con la normativa vigente en Uruguay para la investigación con seres humanos (Decreto CM/515 Poder Ejecutivo, Ley Habeas Data 2009).

\section{Instrumento}

Se utilizó la escala índice de bienestar personal (PWI), en su versión en español adaptada de la versión española (Casas et al., 2011) para Chile (Alfaro et al., 2016). Esta escala mide la satisfacción con la vida a través de un puntaje global, así como la satisfacción en siete ámbitos específicos. El instrumento mostró buenas propiedades psicométricas y estabilidad cultural (The International Wellbeing Group, 2013). El Alfa de Cronbach en estudios internacionales se sitúa en un rango de .70 a .85, en tanto en la versión usada es de .77. La escala consta de siete ítems que corresponden a las áreas: nivel de vida: "Qué tan satisfecho estás con tu nivel de vida", salud: "Qué tan satisfecho estás con tu salud", logros: "Qué tan satisfecho estás con las cosas que has logrado en la vida", relaciones con otros (pares y fami(ia): "Qué tan satisfecho estás con tus relaciones con otras personas", seguridad: "Qué tan satisfecho estás con cuan seguro/a te sientes", conexión con la comunidad: "Qué tan satisfecho estás con los grupos de gente del cual formas parte", y seguridad futura: "Qué tan satisfecho estás con tu seguridad de tu futuro". La escala Likert va de 0 a 10, siendo 0 (completamente insatisfecho) y 10 (completamente satisfecho). El índice se calcula sumando todos los ítems y transformando los puntajes en una escala de 0-100. Se usó una puntuación general de la escala y la media de cada dominio/ítem.

\section{Análisis estadísticos}

Se utilizó el paquete estadístico SPSS Statisctics en su versión 21 (IBM Corporation, 2012). Un valor de $p$ de 0.05 se utilizó como valor estándar de significación estadística, aunque se incluyeron pruebas para un valor de $p<0.01$. 
En primer lugar, se calcularon los estadísticos descriptivos para características demográficas y para el total de la muestra. Seguidamente, se calcularon las diferencias por sexo, lugar de residencia y tramos de edad con relación a cada dominio del PWI. Se analizaron T test independientes y Anova para variables continuas. Para el análisis comparativo entre grupos de edad, se comprobó que la distribución de las variables de cada grupo no sigue una distribución normal, no habiendo varianzas iguales, por tanto, se optó por realizar el test de Games-Howell (prueba post hoc no paramétrica) luego del análisis de Anova. Para esta prueba se calcularon los estadísticos de ETA cuadrado para analizar la medida del tamaño del efecto en más de dos grupos. Seguidamente se calculó el estadístico omega cuadrado para tener una medida poblacional más exacta del tamaño del efecto en los grupos.

\section{Resultados}

Al observar las medidas en cada dominio del PWI, surge que los adolescentes en Uruguay están más satisfechos con el dominio de conexión a la comunidad $(M=87.6 ; D E=1.87)$ y nivel de vida $(M=85.2 ; D E=1.86)$ y menos satisfechos con los dominios de seguridad $(M=76.4 ; D E=2.46)$ y seguridad a futuro $(M=77.4 ; D E=2.36)$ (véase tabla 1$)$.

En cuanto a la comparación entre varones y chicas en cada dominio del PWI, se presentan diferencias significativas en todas las medias de BPS en los siete dominios. Las chicas reportaron menor nivel de BPS en todas las áreas de la vida que indaga el PWI (véase tabla 2).

El análisis de los resultados a la luz de la ubicación geográfica de los jóvenes - ciudad capital o ciudades del interior- no arroja diferencias de significación en ninguno de los dominios del PWI.

Cuando se analiza por tramos de edad (adolescencia temprana 12 a 14 años, media de 15 a 17 y tardía de 18 a 21) se observan diferencias significativas en las medias de todos los dominios, menos en el de conexión a la comunidad (véase tabla 3); en tanto el $\omega^{2}$ reporta un tamaño de efecto bajo. El grupo de adolescencia temprana (12-14) muestra un nivel más elevado de BPS en todos los dominios del PWI en comparación con los adolescentes de mayor edad. Se observa una tendencia decreciente en el nivel de BPS entre los grupos de menor edad y de adolescencia media (15 a $17)$; nivel de BPS que vuelve a aumentar en los adolescentes mayores (18 a 21), a excepción del dominio sobre seguridad

Tabla 1 Índice de bienestar personal PWI N=5189

\begin{tabular}{lcc}
\hline \multicolumn{1}{c}{ Dominios } & Media & DE \\
\hline Salud & 84.8 & 1.86 \\
Nivel de vida & 85.2 & 1.86 \\
Logros & 81 & 2.07 \\
Seguridad & 76.4 & 2.46 \\
Conexión a la comunidad & 87.6 & 1.87 \\
Seguridad futura & 77.4 & 2.36 \\
Relaciones con otros & 83.8 & 2.02 \\
\hline
\end{tabular}

a futuro, que no sigue esta tendencia. Las medias de BPS de este último grupo siguen siendo menores a las de los adolescentes menores (12 a 14). En todos los grupos de edad, el domino en el que informan estar más satisfechos es el de los grupos de pertenencia (conexión a la comunidad) y menos satisfechos con el de seguridad y seguridad a futuro.

\section{Discusión}

El objetivo del presente estudio es obtener un perfil nacional de BPS en adolescentes escolarizados, considerando cada área de la vida, y realizando un análisis por sexo, grupos de edad y lugar de residencia. Los resultados indican un nivel de BPS comparativamente similar al de los hallazgos internacionales -entre 71 a 90 puntos- (The International Wellbeing Group, 2013).

Los adolescentes escolarizados uruguayos están más satisfechos con su pertenencia a los diferentes grupos en su comunidad y menos satisfechos con su seguridad y seguridad a futuro. Estos resultados coinciden con el estudio realizado en el país con jóvenes escolarizados (Fernández et al., 2018). Para los adolescentes, su pertenencia a grupos de pares y grupos en su comunidad es muy importante, lo que podría explicar el alto nivel de BPS en ese dominio específico (conexión a la comunidad y grupos de pertenencia). Estudios antecedentes muestran que los adolescentes presentan un elevado nivel de satisfacción con su entorno (Casas, Bello, González \& Aligue, 2012; Castellá et al., 2012; Fernández et al., 2018; Tonon, Mikkelsen, Rodríguez de la Vega \& Toscano, 2016). El relacionamiento saludable con los pares, el apoyo social y las interacciones de los adolescentes en su vecindario e instituciones, promueven un alto nivel de bienestar, a su vez ofician de factores de protección frente a posibles conductas de riesgo (Fernández et al., 2018; Rodríguez- Fernández., Ramos-Díaz., Ros., Fernández-Zabala., y Revuelta, 2016; Tomyn \& Cummins, 2011). El estudio de Rees y Dinisman (2015) evidenció que, en los 11 países comparados, la relación con amigos, familia y la comunidad era fundamental para el bienestar en estas etapas. El estudio de Leria y Salgado (2019) muestra que el contexto escolar y familiar son variables predictivas de una elevada satisfacción vital en estudiantes de primaria y secundaria. El sentido de pertenencia a la comunidad influye en mejorar y aumentar la valoración positiva que hacen los adolescentes de su propia vida (Guzmán, Alfaro \& Varela, 2018). La relación causal de este dominio con otras variables es un tema a profundizar a futuro; para poder trabajar más en el campo de la protección, lo que requerirá de estudios de tipo longitudinal.

Por otra parte, en nuestro país hay una creciente preocupación de la población sobre seguridad y las estadísticas muestran que las tasas en algunos delitos se han duplicado en el último año (Aboal, Lanzilotta, Domínguez \& Vairo, 2015; Observatorio de Criminalidad y Violencia, MI, 2018). Este aspecto del contexto local podría estar influyendo en el autorreporte de los adolescentes, en cuanto a su bajo nivel de BPS con relación a los dominios de seguridad, tema que ya fuera visualizado en un estudio empírico con estos grupos etarios (Daset et al., 2009) al extraer un síndrome de temor. 
Tabla 2 Bienestar psicológico subjetivo, diferencias de medias según sexo

\begin{tabular}{lccccccc}
\hline \multicolumn{1}{c}{ Dominios PWI } & Varones M & DE & Mujeres M & DE & \multicolumn{2}{c}{ Var. vs. Muj. } & Cohen d \\
& & & & & $\mathrm{t}$ & (df) & \\
\hline Salud & 86.9 & 1.70 & 82.9 & 1.97 & 7.72 & $(5026)^{* *}$ & 2.17 \\
Nivel de vida & 87 & 1.72 & 83.6 & 1.97 & 6.54 & $(4991)^{* *}$ & 1.83 \\
Logros & 82. & 1.96 & 80.1 & 2.16 & 3.26 & $(4947)^{* *}$ & 0.96 \\
Seguridad & 81.5 & 2.10 & 72 & 2.66 & 13.93 & $(4926)^{* *}$ & 3.96 \\
Conexión a la comunidad & 88.5 & 1.73 & 86.8 & 1.97 & 3.33 & $(4972)^{* *}$ & 0.91 \\
Seguridad futura & 79.2 & 2.18 & 75.9 & 2.49 & 4.97 & $(4972)^{* *}$ & 1.41 \\
Relaciones con otros & 85.3 & 1.92 & 82.4 & 2.10 & 5.03 & $(4989)^{* *}$ & 1.44 \\
\hline
\end{tabular}

${ }^{*} p<.05$

${ }^{* *} p<.01$

Tabla 3 Bienestar psicológico subjetivo. Diferencias de medias según tramos de edad

\begin{tabular}{lcccccccccccc}
\hline \multicolumn{1}{c}{ Edades } & \multicolumn{1}{c}{$12 / 14$} & \multicolumn{1}{c}{$15 / 17$} & \multicolumn{1}{c}{$18 / 21$} \\
\multicolumn{1}{c}{$\mathrm{N}=1863$} & \multicolumn{2}{c}{$\mathrm{N}=2523$} & $\mathrm{~N}=803$ & & & & & & \\
\hline Dominios PWI & $\mathrm{M}$ & $\mathrm{DE}$ & $\mathrm{M}$ & $\mathrm{DE}$ & $\mathrm{M}$ & $\mathrm{DE}$ & $\mathrm{Sig}$. & $\mathrm{F}$ & $\mathrm{df}$ & $\eta_{\mathrm{p}}^{2}$ & $\omega^{2}$ \\
Salud & 86.6 & 1.89 & 83.4 & 1.89 & 84.7 & 1.67 & $* *$ & 16.01 & 2 & 0.1 & 0.05 \\
Nivel de vida & 87.7 & 1.85 & 83.7 & 1.88 & 84.1 & 1.77 & $* *$ & 24.94 & 2 & 0.01 & 0.09 \\
Logros & 83.5 & 2.11 & 78.9 & 2.09 & 81.6 & 1.85 & $* *$ & 25.76 & 2 & 0.01 & 0.09 \\
Seguridad & 79.5 & 2.48 & 74.3 & 2.49 & 76.1 & 2.25 & $* *$ & 23.05 & 2 & 0.009 & 0.08 \\
Conexión a la comunidad & 88.2 & 1.93 & 87.2 & 1.84 & 87.5 & 1.81 & & 1.43 & 2 & 0.001 & 0.00 \\
Seguridad futura & 82 & 2.25 & 75.2 & 2.4 & 73.9 & 2.31 & $* *$ & 52.4 & 2 & 0.021 & 0.02 \\
Relaciones con otros & 84.7 & 2.13 & 83 & 1.99 & 84 & 1.84 & $*$ & 3.76 & 2 & 0.001 & 0.01 \\
\hline
\end{tabular}

${ }^{*} p<.05$

${ }^{* *} p<.01$

Segun test Games-Howell

Los resultados multidimensionales del bienestar psicológico subjetivo comparando varones y chicas muestran diferencias significativas según el sexo, siendo las chicas las que reportan un menor nivel de bienestar en todos los dominios. Esto coincide con estudios previos internacionales y los realizados en el país (Brann et al., 2017; Casas et al., 2007; Castellá-Sarriera et al., 2012; Fernández et al., 2018; García et al., 2017; González et al., 2017; Tomyn \& Cummins, 2011; Varela et al., 2018). Las posibles explicaciones brindadas refieren a que, el periodo de desarrollo y las pautas culturales, como el impacto de los estereotipos de género sobre la percepción de bienestar, una mayor presión social y más responsabilidades adjudicadas al rol femenino más tradicional, podrían estar incidiendo en estas diferencias. Se necesitan estudios de tipo longitudinal que permitan acercarnos a un esclarecimiento de las variables que actúan en el fenómeno. La profundización en las diferencias de género en el reporte del BPS, considerando además cada dominio, permite considerar estrategias educativas específicas y promover un saludable desarrollo psicológico en esta etapa etaria (Chen et al., 2019). En cuanto a posibles variaciones en el nivel de BPS, según el lugar de residencia (capital e interior del país), no se encontraron diferencias significativas en ninguno de los dominios. Por tanto, los resultados muestran que, independientemente de la zona, existe homogeneidad en la percepción del bienestar en los adolescentes.

Respecto a las diferencias por tramos de edad, los resultados indicaron que el nivel de BPS es variable en la adolescencia. Los adolescentes menores reportaron mayor nivel de BPS en todos los dominios con relación a sus pares mayores en edad. El grupo de adolescencia media (15 a 17) fue el grupo que mostró niveles más bajos de BPS. Estos resultados coinciden con el estudio nacional anterior (Fernández et al., 2018) y al diferenciar el tramo de adolescencia tardía hasta los 21 años, puede observarse aún mejor la tendencia del nivel de BPS en las transiciones dentro de la propia adolescencia. Estos resultados coinciden con estudios internacionales que evidencian que conforme avanza la edad, el BPS disminuye (Alfaro et al., 2016; Casas \& GonzálezCarrasco, 2019; Casas, González, Figuer \& Malo, 2009; Lin \& Yi, 2018; Petito \& Cummins, 2000; Varela et al., 2018). Las posibles explicaciones a este hallazgo se centran en que los 
cambios frecuentes a los que se enfrentan los adolescentes, tanto emocionales, físicos y también los desafíos sociales y a nivel académico podrían provocar un mayor nivel de estrés y terminar afectando su nivel de BPS (Lin \& Yi, 2018).

Atendiendo a la función protectora que tiene el BPS, según sugieren los estudios de referencia, el diseño y evaluación de políticas públicas orientadas a adolescentes debería tomar en cuenta los indicadores subjetivos de bienestar de esta etapa etaria (Casas, 2011; Noble \& McGrath, 2014). Esta información debería formar parte importante en el diseño de políticas públicas orientadas a jóvenes, por lo que redoblar el esfuerzo en este tipo de investigaciones de campo se hace imprescindible.

El estudio debe considerarse a la luz de ciertas limitaciones; en tanto se basa exclusivamente en el autorreporte de los mismos jóvenes, si bien se plantea que son un agente importante a la hora de informar sobre sus experiencias y percepciones; en el futuro habría que considerar incluir otros informantes (profesores, padres, tutores).

En el ámbito metodológico, también sería beneficioso contar con un diseño longitudinal, que permita evidenciar los posibles procesos de cambio y transiciones en el periodo de la adolescencia, pubertad/adolescencia y adolescencia/ adultez, con seguimiento en el tiempo y en relación con otras variables como las psicopatológicas y las sociodemográficas, incluyendo además poblaciones de zonas rurales.

\section{Agradecimientos}

Los autores desean agradecer al sociólogo Héctor Suárez y a la socióloga Jessica Ramírez de la Junta Nacional de Drogas, Uruguay, por su colaboración con la base de datos.

\section{Contribuciones}

Todos los autores contribuyeron sustancialmente en el estudio.

\section{Conflictos de interés}

No existen conflictos de intereses de ningún tipo.

\section{Financiamiento}

Este estudio no recibió ningún tipo de financiamiento o beca.

\section{Referencias}

Aboal, D., Lanzilotta, B., Domínguez, M., \& Vairo, M. (2015). The cost of crime and violence in five Latin American countries. European Journal on Criminal Policy and Research, 21(3). http:// dx.doi.org/10.1007/s10610-015-9295-5

Alfaro, J., Guzmán, J., García, C., Sirlopú, D., Reyes, F., \& Varela, J. (2016). Psychometric properties of the Spanish version of the personal well-being index-school children (PWI-SC) in chilean school children. Child Indicators Research, 9, 731-742. http:// dx.doi.org/10.1007/s12187-015-9342-2

Brann, E., Chaplin, J., Agelii, M., Sjoberg, A., Nikalsson, A., Albertsoon-Wikland, K., \& Lissner, L. (2017). Declining well-being in young Swedes born in 1990 versus 1974. Journal of Adolescent Health, 60, 306-312. http://dx.doi.org/10.1016/j.jadohealth.2016.10.009
Casas, F., \& González-Carrasco, M. (2019). Subjective wellbeing decreasing with age: New research on children over 8 . Child Development, 90(2), 375-394. http://dx.doi.org/10.1111/cdev.13133

Casas, F. (2011). Subjective social indicators and child and adolescent wellbeing. Child Indicators Research, 4, 555-575. http:// dx.doi.org/10.1007/s12187-010-9093-z

Casas, F., Bello, A., González, M., \& Aligue, M. (2012). Personal wellbeing among Spanish adolescents. Journal of Social Research \& Policy, 3(2), 19-45.

Casas, F., González, M., Figuer, C., \& Malo, S. (2009). Satisfaction with spirituality, satisfaction with religion and personal wellbeing, among Spanish adolescents and young university students. Applied Research in Quality of Life, 4(1), 23-45. http://dx.doi. org/10.1007/s11482-009-9066-X

Casas, F., Figuer, C., González, M., Malo, S., Alsinet, C., \& Subarroca, S. (2007). The well-being of 12 to 16 years old adolescents and their parents from 1999 to 2003 Spanish samples. Social Indicators Research, 83, 87-115. http://dx.doi.org/10.1007/s11205006-9059-1

Castellá-Sarriera, J., Saforcada, E., Tonon, G., Rodríguez de la Vega, R., Mozobancyk, S., \& Bedin, L. (2012). Bienestar subjetivo de los adolescentes: un estudio comparativo entre Argentina y Brasil. Psychosocial Intervention, 21(3), 273-280. http:// dx.doi.org/10.5093/in2012a24

Chen, X., Cai, Z., He, J., \& Fan, X. (2019). Gender differences in life satisfaction among children and adolescents: A meta-analysis. Journal of Happiness Studies. http://dx.doi.org/10.1007/ s10902-019-00169-9

Cummins, R., Li, N., Wooden, M., \& Stokes, M. (2014). A demonstration of set-points for subjective well-being. Journal of Happiness Studies, 15, 183-206. http://dx.doi.org/10.1007/s10902013-9444-9

Cummins, R. A., Eckersley, R., Pallant, J., Van Vugt, J., \& Misajon, R. (2003). Developing a national index of subjective wellbeing: The Australian unity well-being index. Social Indicators Research, 64, 159-190. http://dx.doi.org/10.1023/A:1024704320683

Daset, L. R., López-Soler, C., \& Hidalgo, M. D. (2009). Síndromes empíricos en una población adolescente, evaluados mediante el YSR. Ciencias Psicológicas, III(1), 67-82. http://dx.doi. org/10.22235/cp.v3i1.138

Diener, E., Lucas, R., \& Scollon, C. (2006). Beyond the hedonic treadmill. Revising the adaptation theory of well-being. American Psychological Association, 61(4), 305-314. http://dx.doi. org/10.1007/978-90-481-2350-6_5

Fernández, M. E., Van Damme, L., De Pauw, S., Costa-Ball, D., Daset, L. R., \& Vanderplasschen, W. (2018). The moderating role of age and gender differences in the relation between subjective well-being, psychopathology and substance use in Uruguayan adolescents. Revista Latinoamericana de Psicopatología Fundamental. São Paulo, Brasil, 21(3), 486-510. http://dx.doi.org/10.1 590/1415-4714.2018v21n3p486.5

Fernández, M. E. (2017). Enhancing quality of life and mental health in substance using adolescents (tesis doctoral en Psicología y Ciencias de la Educación). Doctorado conjunto Universidad Católica del Uruguay, Universidad de Gante, Bélgica.

Ferreria-Lima, R. F., \& Araújo de Morais, N. (2018). Bem-estar subjetivo de crianças e adolescentes: revisão integrativa. Ciencias Psicológicas, 12(2), 249-260. http://dx.doi.org/10.22235/ cp.v12i2.1689

García, D., Sagone, E., De Caroli, M., \& Nima, A. (2017). Italian and Swedish adolescents: Differences and associations in subjective well-being and psychological well-being. Peer J, 5 e:26868. http://dx.doi.org/10.7717/peerj.2868

Góngora, V., \& Castro-Solano, A. (2018). Bienestar emocional, psicológico y social y variables demográficas en población adulta de la ciudad de Buenos Aires. Psicodebate, 18(1), 72-83. http:// dx.doi.org/10.18682/pd.v18i1.740 
González-Carrasco, M., Casas, F., Vinas, F., Malo, S., Gras, M., \& Bedin, L. (2017). What leads subjective well-being to change throughout adolescence? An exploration of potential factors. Child Indicators Research, 10, 33-56. http://dx.doi.org/10.1007/ s12187-015-9359-6

González-Carrasco, M., Casas, Malo, S., Vinas, F., \& Dinisman, T. (2017). Changes with age in subjective wellbeing through adolescent years: Differences by gender. Journal of Happiness studies, 18, 63-88. http://dx.doi.org/10.1007/s10902-016-9717-1

Guzmán, J., Alfaro, J., \& Varela, J. (2018). Sense of community and life satisfaction chilean in adolescents. Applied Research Quality Life. http://dx.doi.org/10.1007/s11482-018-9615-2

Huebner, E. S. (1999). A multidimensional investigation of the multidimensionality of children's well-being reports: Discriminant validity of life satisfaction and self-esteem. Social Indicators Research, 46(1), 1-22. http://dx.doi.org/10.1023/A:1006821510832

Huebner, S., Suldo, S., McKnight, C. G., \& Smith, L. C. (2004). Life satisfaction in children and youth: Empirical foundations and implications for school psychologists Psychology in the Schools, 41(1), 81-93. http://dx.doi.org/10.1002/pits.10140

Huebner, E. S. (2004). Research on assessment of life satisfaction of children and adolescents. Social Indicators Research, 66(1-2), 3-33. http://dx.doi.org/10.1023/B:SOCI.0000007497.57754.e3

IBM Corporation. (2012). IBM SPSS Statistics (21). http://www-01. ibm.com/software/es/analytics/spss/.

Lau, A., Cummins, R., \& McPherson, W. (2005). An investigation into the cross-cultural equivalence of the personal well-being index. Social Indicator Research, 72(3), 403-430. http://dx.doi. org/10.1007/s11205-004-0561-z

Leria, F., \& Salgado, J. (2019). El efecto del clima social escolar en la satisfacción con la vida en estudiantes de primaria y secundaria. Revista Educación, 43(1). http://dx.doi.org/10.15517/ revedu.v43i1.30019

Lin, W., \& Yi, C. (2018). Subjective wellbeing and family structure during early adolescence: A prospective study. Journal of Early Adolescence, 1-27. http://dx.doi.org/10.1177/0272431618770785

Ma, C. Q., \& Huebner, E. S. (2008). Attachment relationships and adolescents' life satisfaction: Some relationships matter more to girls than boys. Psychology in the Schools, 45(2), 177-190. http://dx.doi.org/10.1002/pits.20288

Noble, T., \& McGrath, H. (2014). Well-being and resilience in school settings. In Fava y Ruini (Eds.), Increasing psychological well-being in clinical and educational settings (pp. 135-152). Interventions and Cultural Contexts, Chapter 9. http://dx.doi. org/10.1007/978-94-017-8669-0_9

Observatorio de Criminalidad y Violencia, Ministerio del Interior, Uruguay. (2018). Datos de delitos del primer y segundo semestre de 2018. Obtenido de https://www.minterior.gub.uy/index. $\mathrm{php} /$ unicom/noticias/5862-datos-de-delitos-primer-semestre-2018.

Park, N. (2004). The role of subjective well-being in positive youth development. Annals, 591. http://dx.doi.org/10.1177/0002716203260078
Petito, F., \& Cummins, R. (2000). Quality of life in adolescence: The role of perceived control, parenting style, and social support. Behaviour Change, 17(03), 196-207. http://dx.doi.org/10.1375/ bech.17.3.196

Rees, G., \& Dinisman, T. (2015). Comparing children's experiences and evaluations of their lives in 11 different countries. Child Indicators Research, 8, 5-31. http://dx.doi.org/10.1007/s12187014-9291-1

Rodríguez-Fernández, A., Ramos-Díaz, E., Fernández-Zabala, A., Goñi, E., Esnaola, I., \& Goñi, A. (2016). Contextual and psychological variables in a descriptive model of subjective wellbeing and school engagement. International Journal of Clinical and Health Psychology, 16, 166-174. http://dx.doi.org/10.1016/j.ijchp.2016.01.003

Rodríguez-Fernández, A., Ramos-Díaz, E., Ros, I; Fernández-Zabala, E., \& Revuelta, L (2016). Bienestar subjetivo en la adolescencia: el papel de la resiliencia, el autoconcepto y el apoyo social percibido. Suma Psicológica, 23, 60-69. http://dx.doi. org/10.1016/j.sumpsi.2016.02.002

Schnettler, B., Orellana, L., Sepúlveda, J., Miranda, H., Grunert, K., Lobos, G., \& Hueche, C, (2017). Psychometric properties of the Multidimensional Students' Life Satisfaction Scale in a simple of Chilean university students. Suma Psicológica, 24, $97-$ 106. http://dx.doi.org/10.1016/j.sumpsi.2017.06.001

Strelhow, M. R. W., de Oliveira-Bueno, C., \& Câmara, S. G. (2010). Percepção de saúde e satisfação com a vida em adolescentes: diferença entre os sexos. Revista Psicologia e Saúde, 2(2), 42-49.

The International Wellbeing Group. (2013). Personal Wellbeing Index, English Manual ( $5^{\text {th }}$ Edition). The Australian Centre on Quality of Life, Deakin University.

Tonon, G., Mikkelsen, C., Rodríguez de la Vega, L., \& Toscano, W. (2016). Children's views on their lives and well-being in 17 countries: Key messages from each country. Children's worlds.

Tomyn, A., Stokes, M., Cummins, R., \& Dias, P. (2019). A rasch analysis of the personal well-being index in school children. Evaluation \& the Health Professions, 1-22. http://dx.doi. org/10.1177/0163278718819219

Tomyn, A., Weinber, M., \& Cummins, R. (2015). Intervention efficacy among "at risk" adolescents: A test of subjective well-being homeostasis theory. Social Indicators Research, 120, 883-895. http://dx.doi.org/10.1007/s11205-014-0619-5

Tomyn, A., \& Cummins, R. (2011). The subjective wellbeing of high school students: Validating the personal wellbeing index-school children. Social Indicators Research. http://dx.doi. org/10.1007/s11205-010-9668-6

Varela, J. J., Guzmán, J., Alfaro, J., Reyes, F., Sirlopú, D., Benavente, M., ..., \& Yaikin, T. (2018). Un análisis ecológico de predictores claves del bienestar en niñas, niños y adolescentes. En Programa Habilidades para la Vida (Ed.), Apoyando el bienestar en las comunidades educativas (pp. 151-178). Santiago: Programa Habilidades para la Vida. 